\title{
Molecular diagnosis and genetic diversity of tick-borne Anaplasmataceae agents infecting the African buffalo Syncerus caffer from Marromeu Reserve in Mozambique
}

Rosangela Zacarias Machado ${ }^{*}$, Marta Maria Geraldes Teixeira², Adriana Carlos Rodrigues², Marcos Rogério André1, Luiz Ricardo Gonçalves ${ }^{1}$, Jenevaldo Barbosa da Silva ${ }^{1}$ and Carlos Lopes Pereira ${ }^{3}$

\begin{abstract}
Background: Tick-borne diseases (TBDs) are very important in relation to domestic ruminants, but their occurrence among wild ruminants, mainly in the African buffalo Syncerus caffer, remains little known.

Methods: Molecular diagnostic methods were applied to detect Anaplasma marginale, Anaplasma centrale, Anaplasma phagocytophilum, Ehrlichia ruminantium and Ehrlichia chaffeensis in 97 blood samples of African buffalo captured at the Marromeu Reserve in Mozambique. Molecular detection of agents belonging to the family Anaplasmataceae were based on conventional and gPCR assays based on msp5, groEL, 16S rRNA, msp2, pCS20 and vlpt genes. Phylogenetic reconstruction of new Anaplasma isolates detected in African buffalo was evaluated based on msp5, groEL and 165 rRNA genes.

Results: All the animals evaluated were negative for specific PCR assays for A. phagocytophilum, E. ruminantium and E. chaffeensis, but 70 animals were positive for $A$. marginale, showing $2.69 \times 10^{0}$ up to $2.00 \times 10^{5} \mathrm{msp} 1 \beta$ copies $/ \mu \mathrm{l}$. This result overcomes the conventional PCR for A. marginale based on msp5 gene that detected only 65 positive samples. Sequencing and phylogenetic analyses were performed for selected positive samples based on the genes msp5, groEL and 16S rRNA. Trees inferred using different methods separated the $29 \mathrm{msp} 5$ sequences from buffalo in two distinct groups, assigned to A. centrale and A. marginale. The groEL sequences determined for African buffalo samples revealed to be more heterogeneous and inferred trees could not assign them to any species of Anaplasma despite being more related to $A$. marginale and $A$. centrale. The highly conserved $16 \mathrm{~S}$ rRNA gene sequences suggested a close relationship of the new 16 sequences with A. centrale/A. marginale, A. platys and A. phagocytophilum.

Conclusions: Our analysis suggests that different species of Anaplasma are simultaneously present in the African buffalo. To the best of our knowledge, this is the first study that diagnosed Anaplasma spp. in the African buffalo and inferred the taxonomic status of new isolates with different gene sequences. The small fragment of msp5 sequences revealed to be a good target for phylogenetic positioning of new Anaplasma spp. isolates.
\end{abstract}

Keywords: African buffalo, Anaplasma marginale, Anaplasma centrale, Anaplasma platys, Anaplasma phagocytophilum, Genotyping, groEL, msp5, 165 rRNA, Mozambique

\footnotetext{
* Correspondence: zacarias@fcav.unesp.br

'Department of Veterinary Pathology, School of Agrarian and Veterinary

Sciences (FCAV/UNESP), Jaboticabal, SP, Brazil

Full list of author information is available at the end of the article
} 


\section{Background}

Tick-borne agents (TBAs) form one of the main groups of pathogens infecting both domestic and wild ruminants in sub-Saharan Africa and in tropical and subtropical regions. In Mozambique, theileriosis, ehrlichiosis, anaplasmosis and babesiosis are the most important tick-borne diseases (TBDs), causing significant economic losses to the national cattle industry [1].

Wild ruminants may play a role as hosts and reservoirs for several tick-borne pathogens, especially Anaplasmataceae agents and piroplasms. For instance, Anaplasma phagocytophilum, Ehrlichia chaffeensis and Ehrlichia ruminantium are of major concern due to their importance in veterinary and/or human medicine. Anaplasma phagocytophilum is transmitted by ticks of the genus Ixodes and causes tick-borne fever in sheep, goats and cattle in Europe and granulocytic anaplasmosis in humans [2, 3]. This pathogen has been recognized as the causal agent of illnesses in ruminants in Scotland (United Kingdom), Ireland and Scandinavia [4]. Ehrlichia chaffeensis, which is transmitted by the tick Amblyomma americanum in the USA, is the causative agent of human monocytic ehrlichiosis [5]. White-tailed deer (Odocoileus virginianus) are considered the natural reservoirs for both pathogens in wildlife in the USA [4]. In turn, E. ruminantium has been reported in Africa and can be transmitted by ticks of the genus Amblyomma (especially A. variegatum and A. hebraeum). Currently, ehrlichiosis is considered to be one of the most important diseases of domestic ruminants in sub-Saharan Africa [6], with a high mortality rate among susceptible sheep, goats and cattle. Although the African buffalo is considered to act as wild reservoir for this agent, clinical signs and prevalence in this animal species remain little known $[7,8]$.

Although several tick-borne agents (TBA) may affect buffalo, special attention needs to be paid to $A$. marginale, since this is an important pathogen that is responsible for significant economic losses relating to cattle-rearing in South America and Africa [9]. In these regions, this bacterium can be transmitted mechanically by hematophagous dipteran insects, including various species of Tabanus and Stomoxys, and by some mosquito species in the genera Culex and Aedes [10]. Although A. marginale has already been detected in several wild ruminant species, such as Odocoileus virginianus, O. hemionus hemionus, O. hemionus columbianus, Antilocapra americana, Cervus elaphus nelson and Ovis canadensis canadensis in North America and Connochaetes gnou, Damaliscus dorcas phillipsi and Sylvicapra grimmia grimmi in Africa [11], most of the studies on the occurrence, seroepidemiology and molecular characterization of these agents have been conducted among cattle $[12,13]$. When A. marginale infects ruminant species other than cattle, the infection is generally of a chronic nature [11].

In Mozambique, large numbers of African buffalo are maintained in national parks under the protection of the country's legislation. However, there are still no studies on the prevalence of tick-borne pathogens circulating in this group of animals. In the present study Anaplasma species was detected in African buffalo in the Marromeu Reserve (Mozambique) and characterized based on msp5, groEL and $16 S$ rRNA genes for comparison and phylogenetic inferences.

\section{Methods \\ Experimental area}

In 2011, blood samples were collected from 97 African buffalo (Syncerus caffer) in Mozambique, Marromeu Reserve. This reserve is a special buffalo protection area located in the Marromeu district (Sofala Province), with an area of 1,500 $\mathrm{km}^{2}$ (www.jenmansafaris.com). Sampled animals were apparently healthy young male and female individuals. Blood samples had been collected before the animals were transferred from Marromeu Reserve to the Gorongosa Reserve, a distance of $c .300$ kilometers.

\section{Samples and DNA extraction}

Blood samples were collected from the buffalo using EDTA and were mixed (v/v) with ethanol for further DNA extraction. In Brazil, the blood samples from these naturally infected buffalo were incubated in a lysis buffer (1 \% SDS, $100 \mathrm{mM}$ EDTA at pH 8.0, $20 \mathrm{mM}$ Tris- $\mathrm{HCl}$ at $\mathrm{pH} 8.0$ and $350 \mathrm{mg} / \mathrm{ml}$ of proteinase $\mathrm{K}$ ) at $37^{\circ} \mathrm{C}$ for $18 \mathrm{~h}$ and centrifuged at $14,000 \times g$ for $5 \mathrm{~min}$. The DNA was purified using Wizard Purification Systems (Promega). The concentration of each DNA sample was determined in a NanoDrop 2000c spectrophotometer (Thermo Scientific, San Jose, CA, USA).

\section{PCR screening for tick-borne pathogens qPCR}

A quantitative real-time PCR, based on a fragment of msp $1 \beta$ gene of $A$. marginale and previously described by Carelli et al. [14], was used aiming to estimate the parasitemia by means of absolute quantification (number of copies/ $\mu \mathrm{l})$. Additionally, a multiplex qPCR for A. phagocytophilum (msp2 gene) and E. chaffeensis ( $v l p t$ gene) was performed [15]. Serial dilutions of plasmid DNA containing the target sequence were performed aiming to construct standards with different concentrations of the target sequence $\left(2.0 \times 10^{7}\right.$ copies/ $\mu$ lo $2.0 \times 10^{0}$ copies $/ \mu \mathrm{l}$ ) of studied agents. The number of plasmid copies was determined in accordance with the formula $(\mathrm{Xg} / \mu \mathrm{lDNA} /[$ plasmid size $(\mathrm{bp}) \times 660]) \times 6.022 \times 10^{23} \times$ plasmid copies $/ \mu \mathrm{l}$. The amplification reactions were performed using a final total reaction volume of $10 \mu \mathrm{l}$, 
containing a mixture of $1.0 \mu \mathrm{l}$ of sample DNA, $0.2 \mu \mathrm{l}$ of probe, $0.9 \mu \mathrm{l}$ of each primer, $5.0 \mu \mathrm{l}$ of PCR buffer (IQ Multiplex Power Mix ${ }^{\circ}$, BioRad) and $2.0 \mu \mathrm{l}$ of ultra-pure sterile water (Nuclease-Free Water ${ }^{\circ}$, Promega).

\section{Nested PCR}

DNA samples were screened by different conventional PCR assays: a nested PCR for E. ruminantium [16]; two nested PCRs for Anaplasma spp. based on partial sequences of the $16 S$ rRNA gene for detection of $A$. phagocytophilum, A. bovis and A. platys [17], and for A. centrale and A. marginale [18]; a nested PCR for the groEL gene [19-21]; a PCR based on the major surface protein 5 (MSP5) gene $[22,23]$. For these different protocols we followed similar PCR conditions. For the nested PCR, the first reaction was conducted in a final volume of $25 \mu \mathrm{l}$ of the mixture, containing $5 \mu \mathrm{l}$ of genomic DNA, $12.5 \mu \mathrm{l}$ of Taq PCR Master Mix (Qiagen, Madison, USA), $6.5 \mu \mathrm{l}$ of ultra-pure water and $0.5 \mu \mathrm{l}$ of each primer. In the second reaction, a final volume of $25 \mu \mathrm{l}$ of the mixture was used, consisting of $1 \mu \mathrm{l}$ of the product that had been amplified in the first reaction, $12.5 \mu \mathrm{l}$ of Taq PCR Master Mix, $10.5 \mu \mathrm{l}$ of ultra-pure water and $0.5 \mu \mathrm{l}$ of each primer.

Ultra-pure sterile water was used as negative control in all the PCR assays described above. Anaplasma phagocytophilum and E. chaffeensis DNA positive controls were kindly provided by Dr. J. Stephen Dumler (University of Maryland, Baltimore, MD, USA). The Jaboticabal strain of $A$. marginale was used as positive control [GenBank accession number KJ398398]. In order to prevent PCR contamination, DNA extraction, reaction setup, PCR amplification and electrophoresis were performed in separate rooms.

\section{Sequencing and phylogenetic analysis}

The samples that amplified PCR products corresponding to $m s p 5$, groEL and $16 S$ rRNA genes were purified using a Silica bead DNA gel extraction kit (Thermo Scientific, San Jose, CA, USA), in accordance with the manufacturer's recommendations. The purified material was quantified in a Nanodrop spectrophotometer (Thermo Scientific, San Jose, CA, USA). Sequencing was performed on the purified products using the same set of PCR reaction primers, in an automated sequencer (ABI PRISM 3700 DNA Analyzer; Applied Biosystems, CA, USA) at the Biological Resource and Genomic Engineering Center (CREBIO), FCAV, UNESP, Jaboticabal.

For the phylogenetic analysis on $m s p 5$, groEL and $16 S$ rRNA gene sequences determined in this study, different Anaplasma spp. and related species were used.

Three alignments of partial msp5 (351 bp), groEL (520 bp) and $16 S$ rRNA (502 bp) gene sequences were constructed using Clustal X [24] and adjusted manually.
The phylogenetic analyses were carried out using different methods: the neighbour-joining (NJ) algorithm was run in the Mega 4 software [25]; maximum parsimony (MP) and bootstrap analyses were carried out using PAUP* 4.0b10 [26] with 100 replicates of random addition sequence followed by branch swapping (RASTBR), as previously described [27]; and Maximum Likelihood (ML) analyses were performed using RAxML v.2.2.3 [28]. Bayesian Inference (BI) analysis was done using MrBayes on XSEDE (3.2.6) [29] in Cipres Science Gateway [30]. Akaike information criterion was used in Mega 4 [25] to identify the best-fitting model of nucleotide substitution. The phylogenetic analyses using msp5, groEL and $16 S$ rRNA genes were performed with GTR + gamma, GTR + gamma + proportion of invariable sites and TN93 + proportion of invariable sites models, respectively. The first $25 \%$ of the trees from 100,000,000 generations were discarded as 'burn-in'.

\section{Results \\ Diagnostic evaluation of Anaplasma spp. and Ehrlichia spp. in African buffalo blood samples}

Different specific PCR assays were used to detect Anaplasmataceae DNA in blood samples from African buffalo in the Gorongosa National Park, Mozambique. All the 97 blood samples tested were negative in a specific nested PCR for E. ruminantium (based on $p C S 20$ gene) [16] and in a multiplex real-time PCR for E. chaffeensis (based on vlpt gene) and $A$. phagocytophilum (based on $m s p 2$ gene) [15]. Seventy African buffalo (72.2 \%) were shown to be positive for amplification of fragments of the gene msp $1 \beta$ in the real-time PCR specific for A. marginale. Sixty-five African buffalo (67.0 \%) were shown to be positive in the semi-nested PCR for $A$. marginale based on a fragment of the gene msp5. All of the animals that were positive in the semi-nested PCR were shown to be positive in the qPCR, and five animals that were negative in the conventional PCR were shown to be positive in the qPCR (the mean quantification of these animals was $1.32 \times 10^{1}$ DNA copies/ $\mu \mathrm{l}$ of blood). The absolute quantification in the qPCR ranged from $2.69 \times 10^{0}$ to $2.00 \times 10^{5}$ copies of $m s p 1 \beta-A$. marginale DNA per $\mu \mathrm{l}$ of blood.

Out of the 65 samples that were positive for A. marginale using msp-5 diagnostic PCR, only 29 samples were selected for sequencing due to higher intensity of bands in agarose gels.

PCR based on groEL gene revealed 50 (51.5\%) positive samples for Anaplasma; of these, 35 samples were selected for DNA sequencing. We also used two different assays based on $16 S$ rRNA gene aiming the amplification of DNA for A. centrale, A. marginale, A. platys, A. bovis and $A$. phagocytoplilum $[17,18]$. We only identified 16 positive samples for $16 \mathrm{~S}$ rRNA gene using the protocols described above and all samples were sequenced. 
Genetic variability within $A$. centrale and $A$. marginale clades inferred from msp 5 gene sequences

In this study, we sequenced partial msp5 sequences for Anaplasma spp. amplified from wild African buffalo blood samples in Mozambique, East Africa. We determined 29 sequences for the msp5 gene that showed high identity to Anaplasma spp. in a BLAST search in the NCBI website (www.ncbi.nlm.nih.gov). The sequences were aligned with all Anaplasma msp5 gene sequences available in the GenBank database (Fig. 1), including data from the genome project on three samples of $A$. marginale and one sample of $A$. centrale. A set of $18 A$. marginale and one $A$. centrale sequences from North, Central and South America, Asia and Australia was included in the analysis, with the aim of placing the new sequences in the general phylogeny of Anaplasma spp. Anaplasma phagocytophilum was used as the outgroup in all of the tree reconstructions.

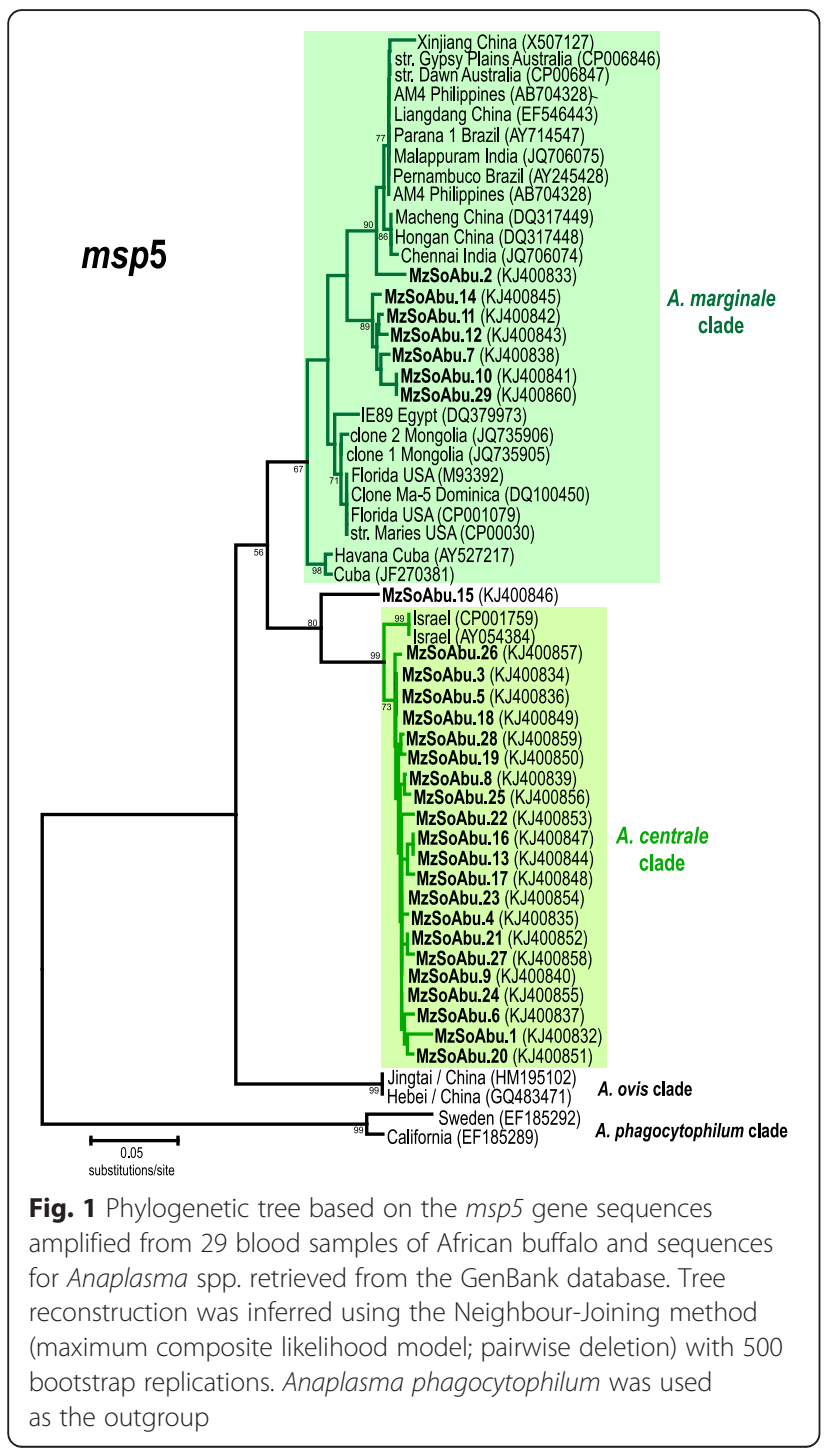

Analyses performed using different methods (Neighbour Joining, Maximum Parsimony, Maximum Likelihood and Bayesian Inference) yielded similar tree topologies and the same relationships for all of the major groups identified in this study; the NJ tree was chosen to represent the relationship observed (Fig. 1). According to the tree topologies inferred using nucleotide sequences (351 bp), the 29 partial msp5 sequences were distributed into two main groups, composed of different isolates of $A$. centrale and $A$. marginale of different geographical provenance. These two species are known to be always more related to each other than to $A$. ovis, and the tree topology inferred here was consistent with previous studies [31], although only partial msp5 sequences ( $351 \mathrm{bp})$ were used in this analysis. Most of the sequences (21 samples) were clustered (with $99 \%$ bootstrap support) with the only two available (and identical) msp5 sequences for $A$. centrale (genome project strain Israel CP001759 and AY054384) and exhibited $1.49 \%$ of genetic divergence within this group. The positioning and high similarity of $m s p 5$ sequences strongly suggest the identification of these 21 samples as isolates of A. centrale. Although this group had the most homogeneous msp5 sequences, the sample from buffalo MzSoAbu.15 was always positioned at the edge of the group ( $80 \%$ bootstrap support). This sample showed approximately $8 \%$ genetic divergence from the $A$. centrale sequences and $10 \%$ from $A$. marginale sequences, and according to the tree topology was most related to the $A$. centrale isolates.

The largest numbers of $m s p 5$ sequences available in the GenBank database are from A. marginale (20 sequences) from isolates with a worldwide distribution (Asia, Australia and South, Central and North America), whose genetic divergences ranged from 0 to $9.8 \%$ (3\% of genetic index of divergences). However, among these $20 \mathrm{msp} 5$ sequences of A. marginale, only the Egyptian sample IE89 (DQ379973) was from Africa. In the present study, a single sample (buffalo MzSoAbu.02) was strongly positioned with $A$. marginale (90\% bootstrap support) and was closely related to isolates of different geographical provenance (North, Central and South America, Asia and Australia). Although positioned together with $A$. marginale msp5 sequences, the sample from buffalo MzSoAbu.02 was the most divergent among the newly-generated sequences and showed divergences ranging from 3.7 to $9.8 \%$ with the sequences available for this species. The comparison of $A$. marginale msp5 sequences retrieved from GenBank and the genome projects showed divergences ranging from 0 to $7.1 \%$; the inclusion of African buffalo samples ranged up to $~ 8.7 \%$.

The remaining six msp5 sequences (MzSoAbu.07, MzSoAbu.10, MzSoAbu.11, MzSoAbu.12, MzSoAbu.14 and MzSoAbu.29) always clustered together (89 \% bootstrap support and $2.3 \%$ genetic divergence) and diverged by $6.5 \%$ from all $A$. marginale sequences (Fig. 1). When these six sequences were added to the calculation of the 
genetic index of internal divergence of $A$. marginale, the values changed from 3.0 to $4.0 \%$. In the current dataset, comprising all the available msp5 sequences from Anaplasma spp., the positioning of these six sequences was always the same, independent of the method used and closeness to $A$. marginale. However, in a reduced dataset, the positioning of these six sequences changed according to the different methods used.

Despite the reduced available data of $m s p 5$ sequences for $A$. centrale, our analysis revealed that there were more heterogeneous sequences of $m s p 5$ among the $A$. marginale sequences (3.0\% internal divergence) than among $A$. centrale sequences ( $2.0 \%$ internal divergence, including the newly-generated sequences). In addition, the genetic divergences between $A$. marginale and $A$. centrale groups were clearly evident and supported their distinction into two different clades. Anaplasma marginale msp5 sequences were separated from A. centrale sequences by a genetic divergence of $14 \%$, from $A$. ovis by $17 \%$ and from $A$. phagocytophilum by $35 \%$. The few available msp5 sequences for other Anaplasma species were for $A$. ovis (two identical sequences from China) and for $A$. phagocytophilum (two sequences from North America and Europe diverging by $4.4 \%$ ).

\section{Polymorphism of groEL sequences close related to $A$. centrale and $A$. marginale}

We generated 35 partial groEL nucleotide sequences $(\sim 520 \mathrm{bp})$ and inferences were made using strains of $A$. marginale, A. centrale, A. ovis and A. phagocytophilum for comparative purposes. The aligned region comprised positions 18 to 537 in the sequence for $A$. marginale St Dawn (CP006847) that was used as guide sequence for groEL gene. Anaplasma centrale and A. marginale groEL sequences retrieved from GenBank showed to be identical in the 520 aligned nucleotide positions, with the exception of A. marginale St Maries from USA $(0.19 \%)$ and $A$. centrale from Rhipicephalus simus from South Africa (0.38 \%) that diverged from each other by $0.58 \%$. The high similarity of groEL sequences between $A$. centrale and $A$. marginale, two recognized distinct Anaplasma species, precluded their separation in the phylogenetic trees inferred (Fig. 2).

Our analysis revealed four heterogeneous sequences from buffalo samples (MzSoAbu.37, MzSoAbu.29, MzSoAbu.24 and MzSoAbu.20) that diverged from each other by $4.0-7.7 \%$. Anaplasma ovis was distantly related to $A$. marginale and $A$. centrale (distance of $20 \%$ ). The above mentioned buffalo samples presented divergence values more closely related to other African buffalo isolates $(2-11 \%)$ than to $A$. ovis (6.6-12.3\%). The high degree of similarity presented by groEL gene sequences associated with the intriguing positioning and the low bootstrap support preclude associating these sequences with neither A. ovis nor A. centrale/A.marginale isolates.

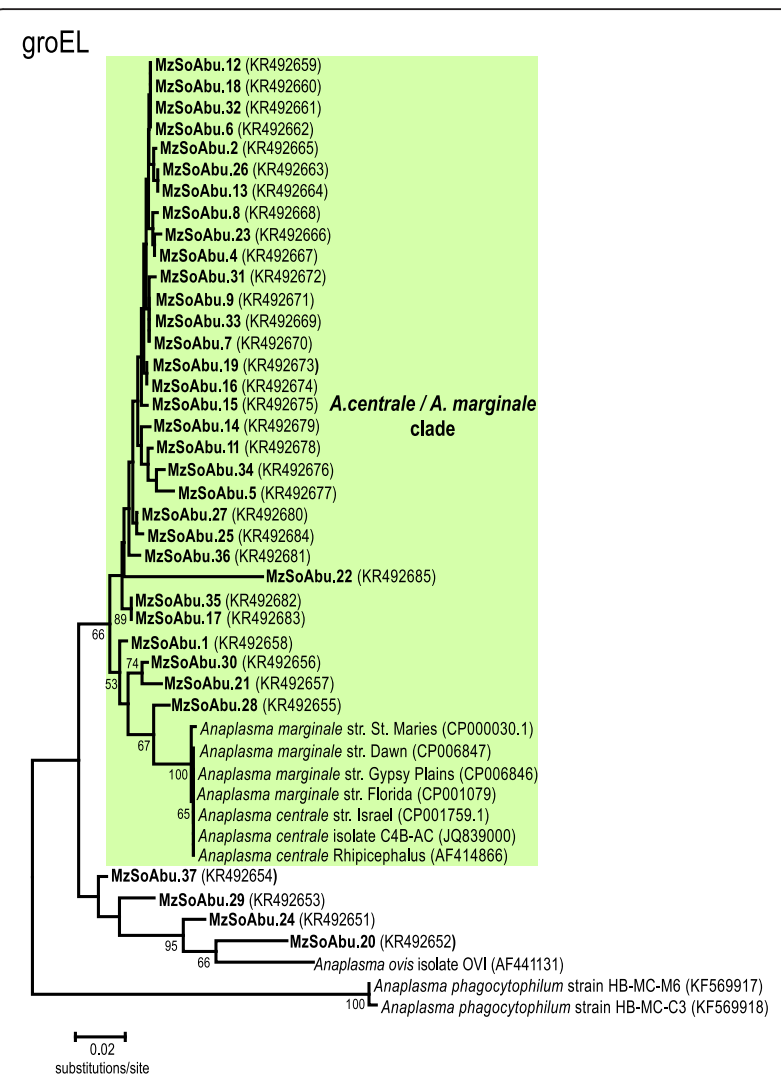

Fig. 2 Phylogenetic relationships of Anaplasma spp. identified in African buffalo blood samples in Marromeu reserve at Mozambique based on the groEL gene. The tree was reconstructed by the Neighbour-Joining method (maximum composite likelihood model; pairwise deletion) with 500 bootstrap replications. Anaplasma phagocytophilum was used as the outgroup; the newly-generated sequences are indicated in bold

Sequences from buffalo samples MzSoAbu.01, MzSoAbu.30, MzSoAbu.21 and MzSoAbu.28 (index of $4 \%)$ showed to be intimately related to the cluster that grouped the clades $A$. marginale and $A$. centrale (divergence ranging between 2-3\%). All of the remaining 27 sequences showed $\sim 3 \%$ of divergence index (ranging from 0.7 to $10 \%)$. The inferences gathered using the groEL sequences retrieved from 35 buffalo samples suggest a closer association to $A$. centrale/A. marginale clade than to other species but makes it difficult to infer the degree of relatedness within the reference strains due to high sequence similarity of the groEL gene. Our analysis showed a high degree of heterogeneity among and within Anaplasma samples evaluated herein (Fig. 2). In addition, we did not observe a congruent phylogeny with inferences using $m s p 5$ gene sequences suggesting that African buffalo may harbor a complex of different strains and/or genotypes of Anaplasma species that we could not detect altogether with $m s p 5$ and groEL genes. 


\section{Analysis of 165 rRNA gene sequences discloses African buffalo infection with $A$. centrale/A. marginale, $A$. platys and $A$. phagocytophylum}

We amplified the $16 S$ rRNA gene sequences from 16 blood samples from African buffalo using different protocols enabling detection of different species of Anaplasma and Ehrlichia [17, 18]. Identity matches after a Blast search revealed five samples similar to $A$. marginale and $A$. centrale (99\% similarity), ten to $A$. platys (98-100\% similarity) and one to $A$. phagocytophilum (100\% similarity). Phylogenies were inferred using both groups of sequences from different protocols and methods of analysis (MP, NJ, ML and BI). All methods employed for inferring $16 S$ rRNA gene trees revealed similar tree tolopogies, with the exception of the Bayesian inference analysis that positioned MzSoAbu.12, MzSoAbu.16 and MzSoAbu.27 in A. centrale/A. marginale clade instead in $A$. platys clade. Three major clades were identified that included sequences from four Anaplasma species: A. phagocytophilum, A. platys, A. centrale and $A$. marginale. Despite the negative results obtained in qPCR specific PCR assay based on msp 2 gene, we identified as $A$. phagocytophilum the sequence from buffalo MzSoAbu.20 that was identical with $A$. phagocytophilum group. Sequences for $A$. platys were related to $A$. phagocytophilum (genetic divergences of $0.8 \%$ ). A total of seven sequences of Anaplasma spp. from African buffalo (MzSoAbu.02, MzSoAbu.04, MzSoAbu.10, MzSoAbu.57, MzSoAbu.93, MzSoAbu.80 and MzSoAbu.95) were positioned with A. platys and two different uncultured Anaplasma spp. (KF010833, KJ831219). A general index of $1 \%$ of genetic divergence was observed in this group and due to the high sequence similarities, some discrepancies were present but showed a better support in the Bayesian inference analysis.

Anaplasma marginale and A. centrale $16 S$ rRNA reference sequences were separated only by $0.2 \%$ and were closely related $(0.24 \%)$ to eight sequences from African buffaloes (MzSoAbu.03, MzSoAbu.05, MzSoAbu.18, MzSoAbu.26 and MzSoAbu.28, MzSoAbu.12, MzSoAbu.16 and MzSoAbu.27) that clustered with both Anaplasma species (Fig. 3).

The $16 S$ rRNA sequences generated in this study and used for Blast search identities presented $\sim 500$ nt but matched only in 154 nucleotide positions in the alignment due to the specificity of protocols for each group of species (A. centrale/A. marginale and A. platys/A. phagocytophilum). The lack of longer sequences to overlap the $\sim 500$ nucleotides determined in this study and the high degree of genetic conservation of the $16 \mathrm{~S}$ rRNA gene sequences may generate weak and unstable tree topologies. In addition, comparison of all sequences available for Anaplasma 165 rRNA gene becomes impossible, unless protocols targeting larger fragments have been used for inferences. In this case, the sensibility of PCR assays might be affected and would not detect efficiently the positive samples. In our study, we allied the specificity of PCR protocols to its use for phylogenetic inferences.

\section{Discussion}

The herd of African buffaloes evaluated in this study presented high molecular prevalence of $A$. marginale and, in some cases, the copy numbers detected in the absolute quantification of msp1 $\beta$ gene fragment was similar to values observed in cattle with acute infection [14]. It is likely that the involvement of other forms of transmission and the capacity to cause persistent infection $[10,13]$ make occurrences of $A$. marginale different from those of the other tick-borne agents.

None of the African buffalo was shown to be positive for A. phagocytophilum, E. chaffeensis and E. ruminantium. However, one sequence showing $0.6 \%$ of divergence with $A$. phagocytophilum was obtained using a nested PCR targeting the $16 S$ rRNA gene, despite the negative results obtained in a specific qPCR PCR based on msp2 gene.

On the other hand, the high prevalence and high numbers of copies of $A$. marginale per $\mu$ l observed in the present study suggest that African buffalo are likely to be infected but also possibly present persistent infection and high levels of parasitemia. Anaplasma marginale presents worldwide distribution on cattle and is the primary cause of economic losses in cattle herds in developing countries [31]. The prevalence of $A$. marginale found in the present study was higher than the $5.4 \%(27 / 500)$ and $17 \%(20 / 116)$ observed among water buffalo in Brazil [32] and Pakistan [33], respectively, but was similar to that found among cattle (79.2 \%) in Mozambique [1]. Even in buffalo presenting low levels of infestation by ticks [34] and high immunological competence [35], A. marginale presented high prevalence under the conditions studied, thus suggesting that this agent might be transmitted by other hematophagous insects that have not yet been identified, which would thus allow high prevalence to become established, even in rural animals.

Data relating to the vector competence and impact of the transmission of $A$. marginale by hematophagous dipterous insects are still scarce. Anaplasma marginale may be transmitted mechanically by different species of tabanids, e.g. Stomoxys calcitrans, and some species of mosquitoes, such as those in the genera Culex and Aedes $[10,36]$. However, even though tsetse flies (Glossina spp.) are common on the African continent and act as competent vectors for important pathogens such as Trypanosoma brucei and Trypanosoma vivax, no studies proving the role of these flies in transmitting $A$. marginale in Africa have been conducted yet. According to Scoles et al. [10], mechanical transmission of $A$. marginale depends on 


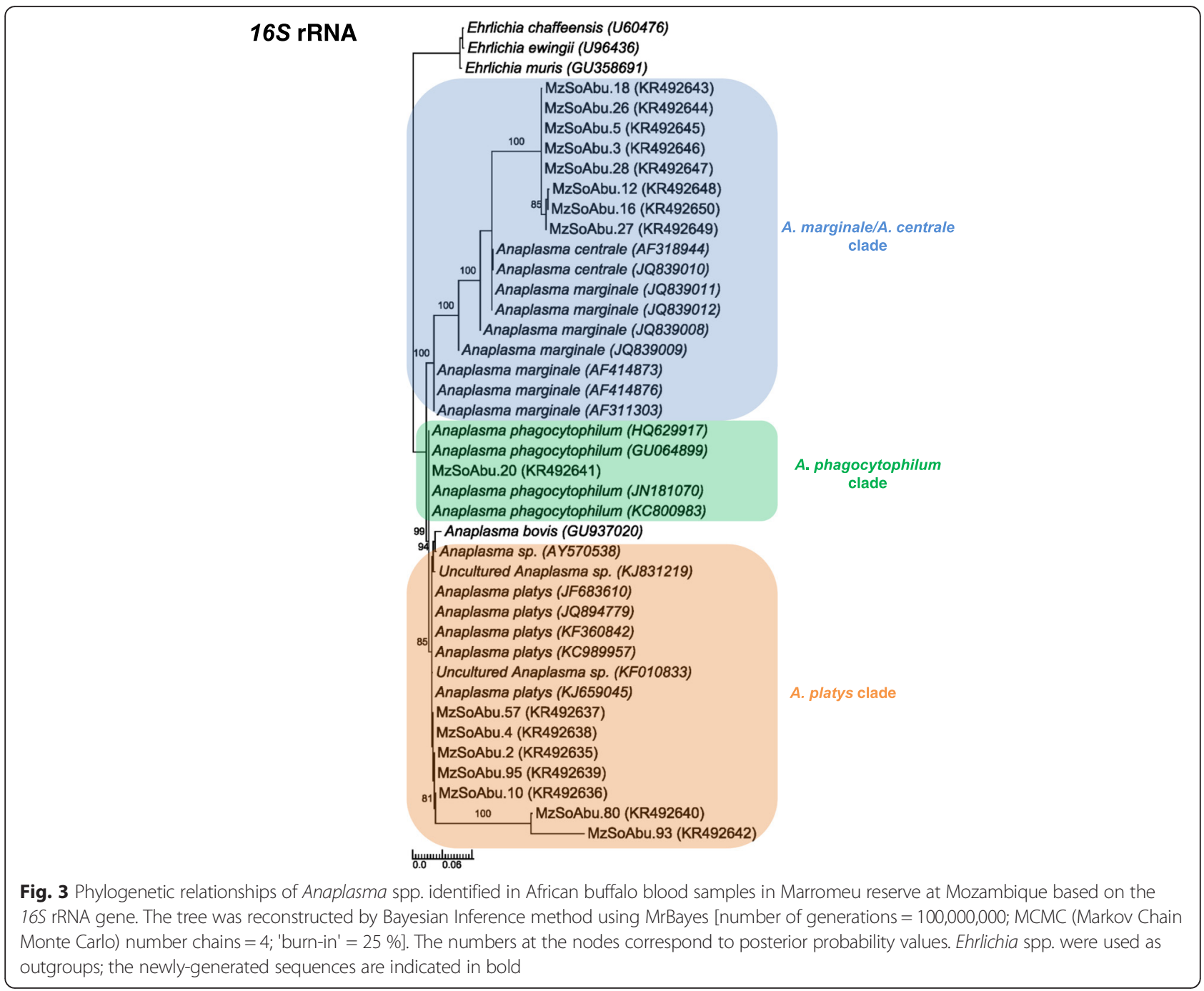

the presence of high levels of parasitemia at the time of the blood meal and is thus limited to herds in which animals are in acute phase of infection. The levels of parasitemia assessed by qPCR absolute quantification (ranging from $2.69 \times 10^{0}$ to $2.00 \times 10^{5}$ copies of $m s p 1 \beta-A$. marginale DNA per $\mu \mathrm{l}$ of blood) presented by African buffalo in Mozambique may facilitate the occurrence of mechanical transmission. Thus, flies may play a role in the transmission of $A$. marginale, given that African buffalo inhabit bush and/or flooded areas to which Rhipicephalus spp. ticks are often poorly adapted. In addition, buffalo may act as wild reservoirs for $A$. marginale, which may become a problem for cattle herds that are kept in areas close to National Reserve areas, given that when cattle come into contact with ticks and flies coming from these buffalo, they may develop clinical disease.

Phylogenies inferred in this study based on msp5, groEL and 165 rRNA gene sequences did not show congruent tree topologies. The 29 Anaplasma spp. msp5 sequences were identified as $A$. centrale and $A$. marginale with a high confidence of bootstrap support and sequence identities. The phylogeny inferred using 35 new Anaplasma spp. groEL sequences did not show congruent topology with those inferred using the $16 \mathrm{~S}$ rRNA and/or msp5 genes from this study. A close association to A. centrale/A. marginale clade was observed for the new 35 groEL sequences but the dataset used in this study did not enable distinguishing even the reference strains from genome projects of both species. Larger fragments or complete gene sequences would help to separate these closely related Anaplasma species. However, the groEL sequences from African buffalo exhibited heterogeneity thus reinforcing the idea that different genotypes and/or species related to Anaplasma circulate among wild animals.

Despite being also conserved, the $16 \mathrm{~S}$ rRNA gene sequences positioned the African buffalo samples in three distinct clades: $A$. platys (7 samples), A. centrale/A. marginale (8 samples) and $A$. phagocytophilum (1 sample). The high specificity of each protocol of $16 \mathrm{~S}$ rRNA gene 
used for diagnosis may not contribute for phylogenetic purposes due to poor data set for alignment construction.

Many of the available $m s p 5$ sequences used in phylogenetic reconstruction dataset were from $A$. marginale isolated from a broad range of localities and hosts (domestic and wild ruminants). The phylogenetic relationships among Anaplasma spp. msp5 sequences inferred in the present study were consistent with the major clades evidenced in previous phylogenies based on $16 \mathrm{~S}$ rRNA, groEL and gltA gene datasets [31].

Very little is known about the circulating species of Anaplasma in wild ruminant hosts in Africa. Considering the divergence among different species and the sequences generated in this study, we can conclude that African buffalo harbor a complex set of genotypes and/ or isolates positioned within the Anaplasma centrale and $A$. marginale groups. In addition to the lack of sequences for some target genes and poor sampling of Anaplasma isolates from different regions, our analysis suggests that protocols for a generic comparison of different genes are needed for a better understanding of tickborne pathogens circulating in wild animals. The small fragment of msp5 sequences (345 bp) was helpful in the identification and phylogenetic positioning of new isolates but need to be reappraised for generic comparative purposes.

Phylogenetic inferences based on the gene msp5 have shown marked similarity between samples of $A$. marginale isolated from cattle and Riphicephalus microplus [31]. Our study using $m s p 5$ gene sequences showed that there is high complexity and distinct genotypes, as yet undescribed, within the Anaplasma centrale and A. marginale circulating in African buffalo. The real impact of these genotypes and/or Anaplasma species on the health of buffalo and cattle in Africa requires further study. There are still insufficient data to prove whether the samples of $A$. marginale circulating in African buffalo are transmitted by biological or mechanical vectors.

A detailed understanding of the genetic diversity and phylogenies of Anaplasma species from different hosts and geographic regions are still required for elucidating the taxonomic and phylogenetic relationships among the currently recognized Anaplasma species, as well as their relationships with other allied species.

\section{Conclusions}

To our knowledge, this is the first molecular study to characterize Anaplasma spp. from African buffalo. We detected a high prevalence of $A$. marginale by means of specific qPCR. A $16 S$ rDNA nested PCR helped to reveal Anaplasma species such as A. phagocytophilum, A. centrale, A. marginale and A. platys but could not render robust and invariable tree topologies for the closest species. The knowledge of new and genetically complex isolates and/or genotypes from Anaplasma species circulating in African buffalo was evaluated with different molecular markers. From our data, a wide range of genetic diversity was recognized among and within Anaplasma species in a very ancient group of wild artiodactyl hosts.

\section{Acknowledgements}

We are grateful to a number of colleagues, students and local people who helped Dr. Carlos Lopes Pereira with the fieldwork for animals capture in the Marromeu Reserve. We would also like to thank Dr. John Stephen Dumler (University of Maryland, Baltimore, MD, USA) for providing the positive controls for Anaplasma phagocytophilum and Ehrlichia chaffeensis.

\section{Funding}

This work was supported by grants from the FAPESP (Fundação de Amparo à Pesquisa do Estado de São Paulo) delivered to RZM and the Brazilian Agency CNPq within the PROAFRICA to MMGT.

\section{Availability of data and materials}

The datasets supporting the conclusions of this article are included within the article. The sequences are submitted in the GenBank database under accession numbers KJ400832-KJ400860 (msp5 gene); KR492651-KR492685 (groEL gene); and KR492636-KR492650 (165 rRNA gene).

\section{Authors' contributions}

RZM planned, designed and supervised the study, and wrote the manuscript; MMGT collected buffalo blood samples and revised the manuscript; ARF performed DNA extraction and phylogenetic analyses, and helped to draft the manuscript; MRA performed molecular techniques and helped to draft the manuscript; LRG performed molecular techniques; JBS performed molecular techniques and helped to draft the manuscript; CLP collected buffalo blood samples. All authors read and approved the final manuscript.

\section{Competing interests}

The authors declare that they have no competing interests.

\section{Author details}

${ }^{1}$ Department of Veterinary Pathology, School of Agrarian and Veterinary Sciences (FCAV/UNESP), Jaboticabal, SP, Brazil. ${ }^{2}$ Institute of Biomedical Sciences, University of São Paulo (ICB/USP), São Paulo, SP, Brazil. ${ }^{3}$ National Admnistration of Conservation Areas, Maputo, Mozambique.

Received: 2 July 2015 Accepted: 19 November 2015

Published online: 17 August 2016

\section{References}

1. Tembue AAM, Silva JB, Silva FJM, Pires MS, Baldani CD, Soares CO, Massard CL, Fonseca AF. Seroprevalence of lgG antibodies against Anaplasma marginale in cattle from south Mozambique. Rev Bras Parasitol Vet. 2011;20:1-7.

2. Stuen S, Granquist EG, Silaghi C. Anaplasma phagocytophilum-a widespread multi-host pathogen with highly adaptive strategies. Front Cell Infect Microbiol. 2013;22:3-31.

3. Bakken JS, Dumler JS. Human granulocytic anaplasmosis. Infect Dis Clin North Am. 2015;29:341-55.

4. Woldehiwet Z. The natural history of Anaplasma phagocytophilum. Vet Parasitol. 2010;167:108-22.

5. Ismail N, Bloch KC, McBride JW. Human ehrlichiosis and anaplasmosis. Clin Lab Med. 2010;30:261-92.

6. Allsopp BA. Natural history of Ehrlichia ruminantium. Vet Parasitol. 2010;167: $123-35$

7. Oberem PT, Bezuidenhout JD. Heartwater in hosts other than domestic ruminants. Onderstepoort J Vet Res. 1987;54:271-5.

8. Peter TF, Burridge MJ, Mahan SM. Ehrlichia ruminantium infection (heartwater) in wild animals. Trends Parasitol. 2002;18:214-8.

9. Kocan KM, de la Fuente J, Guglielmone AA, Meléndez RD. Antigens and alternatives for control of Anaplasma marginale infection in cattle. Clin Microbiol Rev. 2003;16:698-712.

10. Scoles GA, Ueti MW, Palmer GH. Variation among geographically separated populations of Dermacentor andersoni (Acari: Ixodidae) in mid gut 
susceptibility to Anaplasma marginale (Rickettsiales: Anaplasmataceae). J Med Entomol. 2005;42:153-62.

11. Kuttler KL. Anaplasma infection in wild and domestic ruminants: a review. J Wildl Dis. 1984;20:12-20.

12. Cabezas-Cruz A, Passos LMF, Lis K, Kenneil R, Valdés JJ, Ferrolho J, et al. Functional and immunological relevance of Anaplasma marginale major surface protein 1a sequence and structural analysis. Plos One. 2013:8:1-13.

13. Palmer $\mathrm{GH}$, Brayton KA. Antigenic variation and transmission fitness as drivers of bacterial strain structure. Cell Microbiol. 2013;14:1-7.

14. Carelli G, Decaro N, Lorusso A, Elia G, Lorusso E, Mari V, et al. Detection and quantification of Anaplasma marginale DNA in blood samples of cattle by real-time PCR. Vet Microbiol. 2007:124:107-14.

15. Reller ME, Clemens EG, Prakash JA, Dumler JS. Assessment of a quantitative multiplex 5' nuclease PCR for spotted fever and typhus group rickettsioses, Ehrlichia chaffeensis, and Anaplasma phagocytophilum. In: Annals of the 23rd Meeting of the American Society for Rickettsiology. Hilton Head Island; 2009. Abstract no. 51.

16. Faburay D, Geysen B, Munstermann S, Bell-Sakyi L, Jongejan F. Longitudinal monitoring of Ehrlichia ruminantium infection in Gambian lambs and kids by pCS20 PCR and MAP1-B ELISA. BMC Infect Dis. 2007;7:1-11.

17. Massung RF, Slater K, Owens JH, Nicholson WL, Mather TN, Solberg VB, Olson JG. Nested PCR assay for detection of granulocytic ehrlichiae. J Clin Microbiol. 1998;36:1090-5.

18. Ybañez AP, Matsumoto K, Kishimoto T, Inokuma H. Molecular analyses of a potentially novel Anaplasma sp. closely related to Anaplasma phagocytophilum in Sika deer in Japan. Vet Microbiol. 2012;157:232-6.

19. Sumner JW, Nicholson WL, Massung RF. PCR amplification and comparasion of nucleotide sequences from the groESL heat shock operon of Ehrlichia species. J Clin Microbiol. 1997;35:2087-92.

20. Lotric-Furlan S, Petrovec M, Zupanc TA, Nicholson WL, Sumner JW, Childs JE, et al. Human granulocytic ehrlichiosis in Europe: clinical and laboratory findings for four patients from Slovenia. Clin Infect Dis. 1998;27:424-8.

21. Nicholson WL, Castro MB, Kramer VL, Sumner JW, Childs JE. Dusky-footed wood rats (Neotoma fuscipes) as reservoirs of granulocytic ehrlichiae (Rickettsiales: Ehrlichieae) in northern California. J Clin Microbiol. 1999;37:3323-7.

22. Torioni de Echaide S, Knowles DP, McGuire TC, Palmer GH, Suarez CE, McElwain FF. Detection of cattle naturally infected with Anaplasma marginale in a region of endemicity by nested PCR and a competitive enzyme-linked immunosorbent assay using recombinant major surface protein 5. J Clin Microbiol. 1998:36:777-82.

23. Singh $H$, Jyoti Haque M, Singh NK, Rath SS. Molecular detection of Anaplasma marginale infection in carrier cattle. Ticks Tick Borne Dis. 2012;3:55-8.

24. Thompson JD, Higgins DG, Gibson TJ. CLUSTAL W: improving the sensitivity of progressive multiple sequence alignment through sequence weighting, position specific gap penalties and weight matrix choice. Nucleic Acid Res. 1994:22:1673-4680.

25. Tamura K, Peterson D, Peterson N, Stecher G, Nei M, Kumar S. MEGA5: molecular evolutionary genetics analysis using maximum likelihood, evolutionary distance, and maximum parsimony methods. Mol Biol Evol. 2011:28:2731-9.

26. Swofford DL. PAUP*. Phylogenetic Analysis using Parsimony (* and other Methods). Version 4.0b10. Sunderland: Sinauer Associates; 2002.

27. Ferreira RC, Campaner M, Viola LB, Takata CS, Takeda GF, Teixeira MM. Morphological and molecular diversity and phylogenetic relationships among anuran trypanosomes from the Amazonia, Atlantic Forest and Pantanal biomes in Brazil. Parasitol. 2007;134:1623-38.

28. Stamatakis A. RAxML-VI-HPC: maximum likelihood-based phylogenetic analyses with thousands of taxa and mixed models. 2006; Bioinformatics 22, 2688-2690.

29. Ronquist F, Huelsenbeck JP. MrBayes 3: Bayesian phylogenetic inference under mixed models. Bioinformatics. 2003;75:1572-4.

30. Miller MA, Pfeiffer W, Schwartz T. Creating the CIPRES Science Gateway for inference of large phylogenetic trees. New Orleans: Proceedings of the Gateway Computing Environments Workshop (GCE); 2010. p. 1-8.

31. Ybañez AP, Sivakumar T, Ybañez RH, Ratilla JC, Perez ZO, Gabotero SR, et al. First molecular characterization of Anaplasma marginale in cattle and Rhipicephalus (Boophilus) microplus ticks in Cebu Philippines. J Vet Med Sci. 2013;75:27-36
32. Rodríguez SD, Ortiz MAG, Ocampo RJ, Murguía CAV. Molecular epidemiology of bovine anaplasmosis with a particular focus in Mexico. Infect Genet Evol. 2009;9:1092-101.

33. Silva JB, Vinhote WMS, Oliveira CMC, André MR, Fonseca AH, Barbosa JD. Molecular and serological prevalence of Anaplasma marginale in water buffaloes in the northern Brazil. Ticks Tick Borne Dis. 2014;5:100-4.

34. Ashraf QUA, Khan AU, Khattak RM, Ali M, Shaikh RS, Ali M, Qbal F. A report on the high prevalence of Anaplasma sp. in buffaloes from two provincesin Pakistan. Ticks Tick Borne Dis. 2013:4:395-8.

35. Somparn P, Gibb MJ, Markvichitr K, Chaiyabutr N, Thummabood S, Vajrabukka C. Analysis of climatic risk for cattle and buffalo production in northeast Thailand. Int J Biometeorol. 2004;49:59-64.

36. Baldacchino F, Muenworn V, Desquesnes M, Desoli F, Charoenviriyaphap T, Duvallet G. Transmission of pathogens by Stomoxys flies (Diptera, Muscidae): a review. Parasite. 2013;20:1-13.

\section{Submit your next manuscript to BioMed Central and we will help you at every step:}

- We accept pre-submission inquiries

- Our selector tool helps you to find the most relevant journal

- We provide round the clock customer support

- Convenient online submission

- Thorough peer review

- Inclusion in PubMed and all major indexing services

- Maximum visibility for your research

Submit your manuscript at www.biomedcentral.com/submit

) Biomed Central 